\title{
Continuous Deterioration of Glucose Metabolism in Treatment-Naive Male Patients with Human Immunodeficiency Virus and Treated with Tenofovir Plus Lamivudine Plus Efavirenz for 156 Weeks
}

\section{Dafeng Liu ( $\nabla$ ldf312@126.com )}

the Public and Health clinic Center of Chengdu https://orcid.org/0000-0002-6792-641X

Xinyi Zhang

Sichuan University West China Hospital

\section{Ruifeng Zhou}

the Public and Health Clinic Cneter of Chengdu

Lin Cai

the Public and Health Clinic Center of Chengdu

Dongmei Yan

the Public and Health Clinic Center of Chengdu

Lijuan Lan

the Public and Health Clinic Center of Chengdu

Shenghua He

the Public and Health Clinic Center of Chengdu

Hong Tang

Sichuan University West China Hospital

\section{Research}

Keywords: human immunodeficiency virus (HIV), antiretroviral therapy (ART), fasting plasma glucose (PFG), dynamic change, long-term

Posted Date: November 16th, 2021

DOI: https://doi.org/10.21203/rs.3.rs-1056349/v1

License: (1) This work is licensed under a Creative Commons Attribution 4.0 International License.

Read Full License 


\section{Abstract}

Introduction: The dynamic characteristics of glucose metabolism and its risk factors in people living with human immunodeficiency virus (PLWH) accepted primary treatment with the efavirenz (EFV) plus lamivudine (3TC) plus tenofovir (TDF) (EFV+3TC+TDF) regimen are unclear and warrant investigation.

Methods: This study was designed using follow-up study. Sixty-one male treatment-naive PLWH were treated with EFV+3TC+TDF regimen for 156 weeks. The glucose metabolism dynamic characteristics, the main risk factors and the differences among the three CD4+ count groups were analyzed.

Result: In treatment-naive male PLWH who accepted treatment with the EFV+3TC+TDF regimen for 156 weeks, a continuous increase in the fasting plasma glucose (FPG) level, the rate of impaired fasting glucose (IFG) and the glycosylated hemoglobin (HbA1c) level were found. These changes were not due to insulin resistance but rather to significantly reduced islet $\beta$ cell function, according to the homeostasis model assessment of $\beta$ cell function (HOMA- $\beta$ ). Moreover, the lower the baseline CD4+ T cell count was, the higher the FPG level and the lower the HOMA- $\beta$ value. Furthermore the main risk factors for the FPG levels were the $C D 3+C D 8+$ cell count and viral load $(V L)$, and the factors contributing to the HOMA- $\beta$ values were the alanine aminotransferase (ALT) level, $\mathrm{VL}$ and $\mathrm{CD} 3+\mathrm{CD} 8+$ cell count.

Conclusions: These findings provide guidance to clinicians who are monitoring FPG levels closely and are concerned about IFG and decreased islet $\beta$ cell function during ART with the EFV+3TC+TDF regimen for long-term application.

\section{Introduction}

In recent years, there have been sharp increases for those infected human immune deficiency virus (HIV) or those has progressed to acquired immune deficiency syndrome (AIDS). There were about 37.9 million people living with HIV (PLWH), 770,000 people died of HIV-related causes, and 1.7 million people newly infected with HIV worldwide up to the end of $2018 .{ }^{1}$ At October 31,2019 , about 958,000 PLWH were reported nationwide in China, ${ }^{2}$ and by the end of September 30, 2018, 262,442 deaths due to HIV infections had occurred in China. ${ }^{3}$

Antiretroviral therapy (ART) is currently the most effective treatment for AIDS, as it can prolong life expectancy and improve life quality. ${ }^{4-5}$ Studies have shown that a normal life expectancy will be acquired by AIDS patients when their CD4+ count is higher than 350 cells $/ \mathrm{mm}^{3}$ and undetectable levels of the viral load reaches in one year after ART. ${ }^{4-5}$ However, metabolic abnormalities, osteoporosis and cardiovascular diseases have become essential factors affecting the prognosis of AIDS patients. ${ }^{6-9}$

As one of first-line antiretroviral therapy (ART) program since the National Twelfth Five-Year Plan in China the efavirenz (EFV) plus lamivudine (3TC) plus tenofovir (TDF) regimen was used for more than ten years, has a reduced effect on metabolism, and there are few reports about its effect on glucose metabolism in the literature. Our previous study showed that the fasting plasma glucose (FPG) level 
increased within four weeks and then returned to the baseline level at 12 weeks after ART with the EFV plus 3TC plus TDF regimen, especially in patients with CD4+ counts less than 350 cells/ $\mu$ l. However, the dynamic characteristics of glucose metabolism and its contributing factors in such patients are unclear and warrant further examination.

\section{Patients And Methods}

\section{Study Population}

A prospective follow-up cohort study for three-year was conducted with sixty-one male PLWH with treatment-naive who visited the Public and Health Clinic Centre of Chengdu from October 1, 2012, to December $31,2017 .{ }^{10-11}$ The inclusion criteria were as follows: 18 to 65 years old; either sex; HIV-1 antibody positivity according to an enzyme-linked immunosorbent assay with confirmation by Western blotting; CD4+ T cell count $<500$ cells/ $\mu \mathrm{L}$ within 30 days before enrollment; voluntary provision of signed informed consent and agreement to receive follow-up; no plan to relocate from the current address during the trial; and no antiretroviral therapy before the trial. ${ }^{10-11}$

The exclusion criteria were as follows: opportunistic infections or acute infections, malignant tumors related with AIDS at enrollment; opportunistic infections within 3 months of enrollment or an unstable condition within 2 weeks of enrollment; detection of any of the following : hemoglobin lower than $9 \mathrm{~g} / \mathrm{dL}$, white blood cell count lower than $2000 / \mu \mathrm{l}$, neutrophil count lower than $1000 / \mu \mathrm{l}$, platelet count lower than $75000 / \mu \mathrm{l}$, serum creatinine higher than 1.5 times the upper limit of normal (ULN), alanine aminotransferase/alkaline phosphatase/aspartate aminotransferase higher than 3 times the ULN, total bilirubin higher than 2 times the ULN, serum creatine phosphokinase higher than 2 times the ULN, or a creatinine clearance rate lower than $60 \mathrm{~mL} / \mathrm{min}$; current drug use; pregnancy or lactation; severe neurological or mental disease; severe digestive tract ulcers ; and a history of alcoholism. ${ }^{10-11}$ The diagnostic criteria for AIDS, impaired fasting plasma glucose (IFG) and diabetes mellitus (DM) were

obtained from the published guidelines. ${ }^{12-13}$ The patients were divided into three groups based on baseline $\operatorname{CD} 4$ counts $\left(23,12,26\right.$ cases in the $>350,200-350$ and $<200$ cells/ $\mu$ l groups, respectively) ${ }^{4-}$ $5,10-12$

\section{Laboratory indicators test}

At 8:00 am venous blood was drawn from the patients who fasted overnight for at least 12 hours for FPG, fasting serum insulin (FINS), glycosylated hemoglobin (HbA1c), T lymphocyte subsets, and HIV viral nucleic acid (HIVRNA). Glucose oxidase method was used for glucose measurement by the semiautomatic Dias STAT Model 550 analyzer (Bio-Rad) with the kit purchased from Zhejiang Eastern European Biological Products Company. Insulin was tested by electrochemiluminescence immunoassay using an automatic Elecsys2010 analyzer (Roche); HbA1c was tested by high-pressure liquid chromatography using a glycosylated hemoglobin (G7) analyzer (Tosoh Company) with a kit purchased from Bio-Rad. HIV RNA was tested by fluorescence quantitative PCR. A flow cytometer from Beckman 
Coulter was used for the lymphocyte subset parameters (including the CD8+ count, CD4+ count, CD3+ count, $\mathrm{CD} 8+\%, \mathrm{CD} 4+\%$, and $\mathrm{CD} 3+\%)$ measurement. The formulas for calculating the homeostasis model assessment of $\beta$ cell function (HOMA- $\beta$ ) value and the homeostasis model assessment of insulin resistance (HOMA-IR) value were following: $\mathrm{HOMA}-\beta=20 * \mathrm{FINS} /(\mathrm{FPG}-3.5)$ and $\mathrm{HOMA}-\mathrm{IR}=(\mathrm{FPG}$ FINS)/ 22.5) ${ }^{14}$ Before and after accepting ART for $4,8,12,24,36,48,60,72,84,96,108,120,132,144$ and 156 weeks were the follow-up time points. The FPG levels were measured at each follow-up time point, and the FINS and HbA1c levels were detected at $0,12,24,36,48,72,96,120$ and 144 weeks. $^{12}$ Two researchers simultaneously collected, entered and checked the data to ensure data accuracy, authenticity, and integrity.

\section{Patient and Public Involvement}

Patients and the public were not involved in the development of the research questions or in the design of the study. Patients received verbal and written information about the study; however, they were not involved in the recruitment of subjects or the conduct of the study. In addition, the burden of the intervention was assessed by the investigators. After signing an informed consent form, the participants were assessed for eligibility, and data collection was performed. Dissemination of the general results (without personally identifying data) will occur on demand.

\section{Statistical Method}

GraphPad Prism version 8 (GraphPad Inc. US) and Social Sciences software version 17.0(IBM Inc., Armonk, NY, USA) were used. The FPG and FINS levels, HOMA-IR values and HOMA- $\beta$ values had normal distributions, and the statistical analyses were conducted directly. The natural viral load, as indicated by theHIV RNA level, was logarithmically transformed because of abnormal distributions. Themeasured data are expressed as the $\mathrm{x} \pm \mathrm{SD}$ for measurement data, and ANOVA was used for multigroup comparisons, SNK analysis was used for furthercomparison oftwo groups. An independent-sample t-tests was used for comparison of two groups. The comparison for the enumerated data expressed as rate used chi-square test. A statistically significant was pvalue $<0.05$.

\section{Ethical consideration}

The study was approved by the hospital ethics committee of the Public and Health Clinic Centre of Chengdu (PJ-K2012-012-01). All patients gave written informed consent.

\section{Results}

\section{Baseline conditions}

At the Public and Health Clinic Centre of Chengdu from October 1, 2012, to December 31, 2017, sixty-one male PLWH with treatment-naive were enrolled into three groups based on baseline CD4+ counts $(23,12$, 26 cases in the $>350,200$ to 350 , and $<200$ cells/ $\mu$ l groups, respectively). Of them 13 cases of infected by heterosexual contact, 42 cases of infected by homosexual contact, and 5 cases infected by both types of 
sexual contact. The baseline virological and immunological indicators, glucose metabolism indicators and general information of 61 cases are shown in Table $1 .{ }^{10-11}$ At baseline, the HOMA- $\beta$ values in the male PLWH were significantly reduced, with an average of $52.37 \mathrm{mlU} / \mathrm{mmol}$. The HOMA-IR values were slightly elevated, and the average FPG, average FINS and average HbA1c levels were normal (Table $1^{10-}$ $\left.{ }^{11}\right)$. Of them the IFG percentage was $18.03 \%$, and there was no patient with DM.

\section{Dynamic changes of glucose metabolism after ART}

The FPG level in 61 patients (Figure 1A) continuously and gradually increased with prolonged ART, comparing between at each follow-up time point from 84 weeks and at baseline significant difference was found (all $P<0.05$ ), especially at 108,144 and 156 weeks (all $P<0.0001$ ). The HbA1c levels in 61 patients (Figure 1B) slightly decreased over the first 48 weeks and then continuously and gradually increased with prolonged ART, comparing between at each follow-up time point from 48 weeks and at baseline significant difference was found (all $P<0.05$ ), especially from 84 to 156 weeks (all $P<0.0001$ ). The percentage of IFG (Figure 1C) initially quickly increased, peaked at 4 weeks, gradually fell until the trough was reached at 36 weeks, and then increased volatility again until it was measured at 156 weeks, especially from 132 to 156 weeks $(P<0.01)$. There were seven cases with newly diagnosed $\mathrm{DM}$, of them 1 , $1,2,1,2$ cases were diagnosed DM after $4,8,12,72$ and 96 weeks, respectively.

Whereas in 61 patients the FINS level (Figure 2A), HOMA-IR value (Figure 2B), and HOMA- $\beta$ value (Figure 2C) gradually rose to peak values at 48 weeks and then decreased from 48 to 156 weeks, comparing between at each follow-up time point and at baseline significant differences were found of FINS level at $24,36,48,60$ weeks; of HOMA-IR value at 48 weeks, and of HOMA- $\beta$ value at 36 and 48 weeks (all $P<0.05)$.

\section{Long-term influence of the baseline CD4+ count on glucose metabolism after ART}

The lower the baseline CD4+ count was, the higher the FPG levels (Figure $3 \mathrm{~A}$ ) at baseline and at 4, 12 and 96 weeks (all $\mathrm{P}<0.05$ ). In contrast, the lower the baseline CD4+ count, the lower the FINS levels (Figure 3B) from 36 and 144 weeks, the HOMA-IR value (Figure $3 C$ ) at 36 and 48 weeks, and the HOMA- $\beta$ value (Figure 3D) at from 36 to 144 weeks were (all $P<0.05$ ), but the higher the HOMA-IR value (Figure $3 \mathrm{C}$ ) and the HOMA- $\beta$ value (Figure 3D) at 24 weeks was (all $P<0.05$ ).

\section{Risk factors for abnormal glucose metabolism}

By spearman correlation analysis, nonalcoholic fatty liver disease (NAFLD), age, body fat percentage, body mass index (BMI), lean body mass, body fat, the aspartate aminotransferase (AST) level, the alanine aminotransferase (ALT) level, the alkaline phosphatase (ALP) level, the $y$-glutamyl transpeptidase (GGT) level, the $C D 3+C D 8+$ percentage and the serum creatinine $(\mathrm{Cr})$ level were all positively correlated, while $C D 3+C D 8+$ count, the $C D 3+$ count, $C D 3+$ percentage, $C D 3+C D 4+$ count, viral load $(V L)$ and 
immunoglobulin M (IgM) level were negatively correlated with the FPG level (Table 2). By multiple stepwise regression analysis the $C D 3+C D 8+$ cell count and $V L$ were main factors associated with the FPG level (Table 3).

According to Spearman correlation analysis, follow-up duration, the ALT level, the AST level, the GGT level, the ALP level, the CD3+CD4+ percentage, and the ratio of the CD3+CD4+ count to the CD3+CD 8+ count was positively correlated, while the serum cystatin C (Cysc) level, CD3+CD 8+ count and percentage, and VL were negatively correlated with the FINS level (Table 2). By multiple stepwise regression analysis, the ALT level, follow-up duration, $\mathrm{VL}$, and $\mathrm{CD} 3+\mathrm{CD} 8+$ count were the main factors associated with the FINS level (Table 3).

According to Spearman correlation analysis, the ALP level, the Cr level, the uric acid (UA) level and the CD3+CD4+ percentage were positively correlated, while the group stratified by baseline CD3+CD4+ count and VL were negatively correlated with the HOMA-IR values (Table 2). By multiple stepwise regression analysis, the ALT level and VL were the main factors associated with the HOMA-IR value (Table 3).

According to Spearman correlation analysis only the UA level and CD3+CD4+ percentage were positively correlated with the HOMA- $\beta$ value, and none of the anthropometric parameters or biochemical, immunological and virological indicators were negatively correlated with the HOMA- $\beta$ value. By multiple stepwise regression analysis, the ALT level, $\mathrm{VL}$ and $C D 3+C D 8+$ count were the main factors associated with the HOMA- $\beta$ value (Table 3 ).

\section{Discussion}

Our previous studies found that islet $\beta$ cell dysfunction is common even in young male PLWH with normal weight and normal glucose tolerance (NGT) and without significant IR. The EFV plus 3TC plus TDF regimen can lead to glucose impairment in the short term. ${ }^{15}$ This study purpose was identified the dynamic characteristics of glucose metabolism and its contributing factors after ART. We found that in male PLWH after ART, glucose metabolism continuously deteriorated, as indicated by the gradual increase in the FPG level, whereas the IFG rate increased rapidly, followed by a gradual decrease and then another gradual increase. The average FPG level was $6.15 \mathrm{mmol} / \mathrm{L}$ at baseline and increased to 6.33 $\mathrm{mmol} / \mathrm{L}$ at 96 weeks. The $\mathrm{HbA} 1 \mathrm{c}$ level was $5.35 \%$ at baseline and gradually increased to $5.90 \%$ at 156 weeks, but the percentage of IFG was $18.03 \%$ at baseline and volatically increased to $45 \%$ at 156 weeks. There were seven patients with newly diagnosed DM within 96 weeks, and the prevalence of newly diagnosed DM was $11.47 \%$ (7/61) of this cohort, slightly higher than $11.2 \%$, the overall prevalence of the general population in China. ${ }^{13}$ But the proportion of PLWH with prediabetes who progressed to diabetes reached $63.63 \%$ within two years, and the progress rate was $31.815 \%$ annually, higher than literature reported that $25.00 \%$ of individuals with prediabetes who progress to type $2 \mathrm{DM}$ in three to five years. ${ }^{15}$ These changes reflected the continuous deterioration of glucose metabolism. 
There have been some reports in the literature about the prevalence of abnormal glucose metabolism in patients with HIV/AIDS after ART. There have been no reports about the long-term dynamics in glucose metabolism after ART with a specific regimen. One cross-sectional study on the DM among PLWH with ART-naive in Guinea-Bissau showed that the prevalence of DM was 5.8\% (52/893) and the prevalence of IFG was $5.6 \%(50 / 893) .{ }^{16}$ Another cross-sectional study of risk factors for IFG or DM among PLWH in Zambia receiving long-term combined antiretroviral treatment showed that ten percent (26/270) had IFG and 5\% (14/270) had DM. ${ }^{17}$ A large-sample epidemiological study showed that $24.8 \%$ of $262 \mathrm{PLWH}$ and $38.2 \%$ of 1583 PLWH receiving ART treatment had elevated FPG levels ${ }^{18-19}$ and that $2.1 \%$ of 1095 PLWH and $7.1 \%$ of 425 PLWH had DM. ${ }^{20-22}$ The annual increase in the prevalence of DM in PLWH was $4.1 \%$, with a 2.27-fold higher prevalence in 2011 than in 1999, while in the non-HIV-positive population, the annual increase was $3.9 \%$, which represented a 1.62 -fold increase in the prevalence. ${ }^{23}$ Follow-up cohort study results also showed that the crude prevalence of DM ranged from 10.8 to 13.7 per 1000 patient annual, ${ }^{24-26}$ and the pooled prevalence of prediabetes was 125 per 1000 patient annual. ${ }^{26}$ The most important risk factors for prediabetes and diabetes were a diabetes family history, aging, Hispanic heritage or Black, obesity/overweight, lipodystrophy, central obesity, metabolic syndrome, dyslipidemia, treatment with certain ART regimens, and increased baseline FPG ${ }^{24-30}$ No reports about the long-term dynamic characteristics of glucose metabolism after ART with a specific regimen were found in the literature.

In the general population abnormal glucose metabolism is associated

with insulin resistance and impaired islet beta cell function. The study

results showed that in male PLWH, the HOMA-IR value first increased to a peak of $2.03 \mathrm{Um} \mathrm{m}^{\star} \mathrm{mmol} / \mathrm{L}^{2}$ at 48 weeks and then decreased until it was measured again at 156 weeks. The HOMA-IR value was always lower than the reference value of $2.3 \mathrm{Um} * \mathrm{mmol} / \mathrm{L}^{2}$, which indicates insulin resistance. The baseline HOMA- $\beta$ value was $52.37 \mathrm{Um} / \mathrm{mmol}$, which was almost half of the reference value of $100 \mathrm{mlU} / \mathrm{mmol}$, and the peak HOMA- $\beta$ value was $74.30 \mathrm{Um} / \mathrm{mmol}$ at 36 weeks, which was also clearly lower than the reference value. Therefore, the continuous deterioration of glucose metabolism was not caused by insulin resistance but rather by significantly decreased islet $\beta$ cell function.

Our studies have also shown that in male PLWH, the lower the baseline CD4+ count, the lower the HOMAIR value and HOMA- $\beta$ value were during the entire 3-year follow-up period. No reports of the impacts of the baseline CD4+ count on the HOMA-IR value and HOMA- $\beta$ value after long-term ART with a specific regimen were found in the literature.

In this study, it was also demonstrated that age, follow-up duration, NAFLD, most of the biochemical indicators, and the $C D 3+C D 8+$ percentage values had positive correlations, while the CD3+CD4+ cell count and virological indicators had negative correlations with the FPG level. The main factors associated with the FPG level were the CD3+CD8+ cell count and VL. Although none of anthropometric parameters or the biochemical, virological and immunological indicators had a direct correlation with the 
HOMA- $\beta$ values representing islet $\beta$ cell function, the ALT level, VL and CD3+CD $8+$ cell count were the main factors associated with the HOMA- $\beta$ value.

Although CD4+ $T$ cell is recognized for assessing immune, there was an association not between glucose metabolism parameters and CD4+ count, but between glucose metabolism parameters and CD8+ count.

A previous research found that the $\mathrm{CD} 8+$ infiltration into adipose tissue promoted the macrophages recruitment, ${ }^{31}$ and resulted local TNF-a ,other inflammatory mediators and IL- 6 increasing which acted on adipocyte surface receptors and by other mechanisms to reduce insulin receptor substrate-1 (IRS-1), glucose transporter type 4 (GLUT4) and phosphoinositide 3-kinase p85a expression thereby inhibiting insulin signaling, ${ }^{31-36}$ leading to islet beta cell dysfunction and insulin resistance, hyperglycemia.

Some reports stated that FPG levels are inversely and significantly correlated with NADH dehydrogenase (C1) enzyme activity in oxidative phosphorylation; ${ }^{30}$ youth infected with HIV and with IR have lower levels markers of mitochondrial respiration than those without IR. Mitochondrial respiration dysfunctional may contribute to IR in the population. ${ }^{37} \mathrm{HIV}$ infection can cause insulin sensitivity decline, and the replication of HIV has important effects on accessory proteins (Tat, Vpr) and may also cause insulin resistance. Vpr affects insulin transcription by inhibiting PPAR-gamma activity, whereas Tat activates nuclear factorkappa B, inhibits insulin receptor signal translocation, and reduces GLUT4 translocation and phosphorylated insulin receptor-1 (IRS-1) expression, ${ }^{38}$ which leads to insulin resistance, islet beta cell dysfunction and elevated plasma glucose levels.

Because of the lack of female patients who met the inclusion criteria during the enrollment duration of the study from October 1, 2012 to December 31, 2013. Only sixty-one male PLWH with treatment-naive was enrolled in this study and the results only reported for males.

This study first reports dynamic effects of the EFV plus 3TC plus

TDF regimen and the baseline CD4+ count on the glucose metabolism parameters in male PLWH treated with a specific ART regimen. The biochemical, anthropometric parameters, virological and immunological indicators associated with the glucose metabolism parameters were identified. The results showed that in male PLWH initially treatment with the EFV plus 3TC plus TDF regimen for 3 years, the FPG level increased continuously and gradually, the percentage of IFG and HbA1c level also increased gradually, there was no obvious insulin resistance, and there was significantly reduced islet $\beta$ cell function. The main factors associated with the FPG level were the CD3+CD8+ cell count and VL, while those associated with the HOMA- $\beta$ value were the ALT level, VL and CD3+CD8+ count.

The limitations of this study were small sample size, a single-center cohort study, only male patients and only examined the effect of the EFV plus 3TC plus TDF regimen. A large-sample, multicenter study of both male and female patients treated with additional ART regimens and a randomized controlled clinical trial are needed. 


\section{Conclusions}

These findings provide guidance for clinicians who wish to monitor FPG levels closely and who are concerned about islet $\beta$ cell dysfunction and IFG rate during long-term ART with the EFV plus 3TC plus TDF regimen; the focus is on the avoidance of the application of insulin secretagogues and protecting islet $\beta$ cell function when hypoglycemic therapy is needed.

\section{Abbreviations}

AIDS, acquired immune deficiency syndrome. ALT, alanine aminotransferase. ART, antiretroviral therapy. BMI, body mass index. Cr, serum creatinine. Cysc, serum cystatin C. DM, diabetes mellitus. EFV, efavirenz. FINS, fasting serum insulin. FPG, fasting plasma glucose. GGT, y-glutamyl transpeptidase. HbA1c, glycosylated hemoglobin. TDF, tenofovir. 3TC, lamivudine. HOMA- $\beta$, homeostasis model assessment of $\beta$ cell function. HOMA-IR, homeostasis model assessment of insulin resistance. HIV, human immunodeficiency virus. HIV RNA, human immunodeficiency viral nucleic acid. IFG, impaired fasting plasma glucose. NAFLD, nonalcoholic fatty liver disease. ULN, upper limit of the normal value. PLWH, people living with human immunodeficiency virus. VL, viral load.

\section{Declarations}

\section{Ethics approval and consent to participate}

The study was approved by the hospital ethics committee of the Public and Health Clinic Centre of Chengdu (PJ-K2012-012-01). All patients gave written informed consent.

\section{Consent for publication}

All of participants understand that the information will be published without their child or ward's/their relative's (circle as appropriate) name attached, but that full anonymity cannot be guaranteed. All of participants understand that the text and any pictures or videos published in the article will be freely available on the internet and may be seen by the general public. The pictures, videos and text may also appear on other websites or in print, may be translated into other languages or used for commercial purposes. All of participants have been offered the opportunity to read the manuscript.

\section{Availability of data and materials}

All data, models, or code generated or used during the study are available from the corresponding author by request: Dafeng Liu, E-mail: liudf312@126.com

\section{Competing interests}


The authors declare that they have no competing interests.

\section{Funding}

This research was supported by the Twelfth Five-Year Project on Tackling Key Problems of National Science and Technology (2012ZX10001-003), the Sichuan Province Health Commission (130430;17PJ070), and the Chengdu Municipal Health Commission (2019079).

\section{Authors' contributions}

Concept and design: Dafeng Liu, Xinyi Zhang, Ruifeng Zhou, Lin Cai, Dongmei Yan, Lijuan Lan, Shenghua He and Hong Tang; data acquisition: Dafeng Liu, Xinyi Zhang, Ruifeng Zhou, Lin Cai, Dongmei Yan, Lijuan Lan; data analysis and interpretation: Dafeng Liu, Xinyi Zhang, Ruifeng Zhou, Lin Cai, Dongmei Yan, Lijuan Lan; drafting of the manuscript: Dafeng Liu, Xinyi Zhang, Ruifeng Zhou, Lin Cai, Dongmei Yan, Lijuan Lan; administrative, technical, or material support: Dafeng Liu, Ruifeng Zhou, Lin Cai, Dongmei Yan, Lijuan Lan, Shenghua He and Hong Tang; study supervision: Shenghua He and Hong Tang.

\section{ORCID}

Dafeng LiuiDhttps://orcid.org/0000-0002-6792-641X

\section{Acknowledgements}

We would like to thank Dr. Yin Wang in the infectious disease department at the Public and Health Clinic Centre of Chengdu.

\section{A statement about the manuscript in research square}

This manuscript titled as "Continuous deterioration of glucose metabolism in male patients living with human immunodeficiency virus and primary treatment with the tenofovir plus lamivudine plus efavirenz regimen: a 2-year follow-up study" was once submitted to "Diabetology \& Metabolic Syndrome", presented as preprint in the research sqaure according to the following link: https://www.researchsquare.com/article/rs-613518/v1, it has been withdrawn. Now this manuscript titled as "Continuous deterioration of glucose metabolism in treatment-naive male patients with human immunodeficiency virus and treated with tenofovir plus lamivudine plus efavirenz for 156 weeks" was resubmitted to "Diabetology \& Metabolic Syndrome".

\section{References}

1. World Health Organization. HIV/AIDS (2019). https://www.who.int/zh/news-room/factsheets/detail/hiv-aid 
2. Disease Control and Prevention Bureau of National Health Commission of the People's Republic of China. New progress was made in the prevention and treatment of AIDS in China in 2019.http://www.nhc.gov.cn/jkj/s3586/201911/c2388ce70bdd404ea6dfcd886591784d.shtml

3. Huajing Information Network. In 2019, the number of AIDS cases, deaths and the status of anti-AIDS drugs in China are still facing huge challenges. https://www.huaon.com/story/469227

4. Samji H, Cescon A, Hogg RS, et al. Closing the gap: increases in life expectancy among treated HIVpositive individuals in the United States and Canada. PLOS One 2013;8: e81355

5. May MT, Gompels M, Delpech V, et al. Impact on life expectancy of HIV-1 positive individuals of CD4+ cell count and viral load response to antiretroviral therapy. AIDS 2014;2 8:1193-202

6. Samaras K, Wand H, Law M, et al. Prevalence of metabolic syndrome in HIV-infected patients receiving highly active antiretroviral therapy using International Diabetes Federation and Adult Treatment Panel III criteria. Diabetes Care 2007; 30:113-115

7. Stanley TL, Grinspoon SK. Body compositiom and metabolic changes in HIV-infected patients. J Infect Dis 2012; 205: S383-90.

8. Adelzon A. Paula, Mauro Schechter, SuelyH. Tuboi, et al. Continuous Increase of Cardiovascular Diseases, Diabetes, and Non-HIV Related Cancers as Causes of Death in HIV-Infected Individuals in Brazil: An Analysis of Nationwide Data. Plos 2014; 9:1-5

9. Muyanja D, Muzoora C, Muyingo A, Muyindike W, Siedner MJ. High Prevalence of Metabolic Syndrome and Cardiovascular Disease Risk Among People with HIV on Stable ART in Southwestern Uganda.AIDS Patient Care STDS 2016;30:4-10.

10. Liu D, Zhang X, He S, et al. Continuous deterioration of glucose metabolism in male patients living with human immunodeficiency virus and primary treatment with the tenofovir plus lamivudine plus efavirenz regimen: a 2-year follow-up study. Research Square preprint https://www.researchsquare.com/article/rs-613518/v1.

11. Liu D, Zhang $X$, Kang J, et al. Gradual increasing dyslipidemia in treatment-naive male patients with human immunodeficiency virus and treated with tenofovir plus lamivudine plus efavirenz for three years. Diabetol Metab Syndr. 2021 (accepted, DIMS-D-21-00247).

12. AIDS Group, Society of Infectious Diseases, Chinese Medical Association. Third Edition of AIDS Diagnosis and Treatment Guidelines (2015 Edition). Chinese Journal of Clinical Infectious Diseases 2015;8: 385-401

13. Diabetes Society of Chinese Medical Association. Chinese Guidelines for the Prevention and Treatment of Type 2 Diabetes (2013 edition). Chinese Journal of Diabetes 2014;30: 893-942

14. Wallace TM, Levy JC, Matthews DR. Use and abuse of HOMA modeling. Diabetes Care. 2004 Jun;27(6):1487-95

15. Hostalek U. Global epidemiology of prediabetes-present and future perspectives. Clinical Diabetes Endocrinology 2019;5(5)

16. Steiniche D, Jespersen S, Erikstrup C, Krarup H, Handberg A, et al. Diabetes mellitus and impaired fasting glucose in ART-naïve patients with HIV-1, HIV-2 and HIV-1/2 dual infection in Guinea-Bissau: 
a cross-sectional study. Trans R Soc Trop Med Hyg 2016 Apr;110(4):219-27.

17. Shankalala P, Jacobs C, Bosomprah S, Vinikoor M, Katayamoyo P, Michelo C. Risk factors for impaired fasting glucose or diabetes among HIV infected patients on ART in the Copperbelt Province of Zambia. J Diabetes Metab Disord 2017; 16:29.

18. Beraldo RA, Santos APD, Guimarães MP, Vassimon HS, Paula FJA, et al. Body fat redistribution and changes in lipid and glucose metabolism in people living with HIV/AIDS.Rev Bras Epidemiol 2017;20:526-536.

19. Alvarez C, Salazar R, Galindez J, et al. Metabolic syndrome in HIV-infected patients receiving ARV therapy in Latin America.Braz J Infect Dis 2010;14:256-63

20. Mayanja BN, Kasamba I, Levin J, Namakoola I, Kazooba P, et al. COHORT PROFILE: The Complications of Long-Term Antiretroviral Therapy study in Uganda (CoLTART), a prospective clinical cohort.AIDS Res Ther 2017;14:26.

21. Nazisa Hejazi1, Roslee Rajikan1, Christopher Lee Kwok Choong, et al. Metabolic abnormalities in adult HIV infected population on antiretroviral medication in Malaysia: a cross-sectional survey. BMC Public Health 2013; 13:758-69

22. Ataro Z, Ashenafi W, Fayera J, Abdosh T. Magnitude and associated factors of diabetes mellitus and hypertension among adult HIV-positive individuals receiving highly active antiretroviral therapy at Jugal Hospital, Harar, Ethiopia.HIV AIDS (Auckl) 2018; 10:181-192.

23. Oscar H.Divala, Alemayehu Amberbir, Zahra Ismail, et al. The burden of hypertension, diabetes mellitus, and cardiovascular risk factors among adult Malawians in HIV care: consequences for integrated services. BMC Public Health 2016; 16:1243-1254

24. Han WM, Jiamsakul A, Kiertiburanakul S, Ng OT, Sim BL, et al. Diabetes mellitus burden among people living with HIV from the Asia-Pacific region.J Int AIDS Soc 2019;22: e25236.

25. Karamchand S, Leisegang R, Schomaker M, Maartens G, Walters L, et al. Risk Factors for Incident Diabetes in a Cohort Taking First-Line Nonnucleoside Reverse Transcriptase Inhibitor-Based Antiretroviral Therapy.Medicine (Baltimore) 2016;95: e2844.

26. Nansseu JR, Bigna JJ, Kaze AD, Noubiap JJ. Incidence and Risk Factors for Prediabetes and Diabetes Mellitus Among HIV-infected Adults on Antiretroviral Therapy: A Systematic Review and Meta-analysis.Epidemiology 2018;29:431-441.

27. Adelzon A. Paula1, Mauro Schechter2, Suely H. Tuboi3, et al. Continuous Increase of Cardiovascular Diseases, Diabetes, and Non-HIV Related Cancers as Causes of Death in HIV-Infected Individuals in Brazil: An Analysis of Nationwide Data. Plos 2014; 9:1-5

28. Wand H, Calmy A, Carey DL, Samaras K, Carr A, Law MG, on behalf of the INITIO Trial International Coordinating Committee, et al. Metabolic syndrome, cardiovascular disease and type 2 diabetes mellitus after initiation of antiretroviral therapy in HIV infection. AIDS 2007; 21:2445-53.

29. Idiculla J, Ravindra'n GD, D’Souza J, Singh G, Furruqh S. Diabetes mellitus, insulin resistance, and metabolic syndrome in HIV-positive patients in South India. Int J Gen Med 2011; 4:73-78 
30. Sharma TS, Jacobson DL, Anderson L, Gerschenson M, Van Dyke RB, et al. Short communication: The relationship between mitochondrial dysfunction and insulin resistance in HIV-infected children receiving antiretroviral therapy.AIDS Res Hum Retroviruses 2013; 29:1211-7.

31. Nishimura S, Manabe I, Nagasaki M, Eto K, Yamashita H, et al. CD8+ effector T cells contribute to macrophage recruitment and adipose tissue inflammation in obesity. Nat Med 2009; 15:914-20

32. Damouche A, Lazure T, Avettand-Fenoel V, Huot N, Dejucq-Rainsford N, et al. Adipose tissue is a neglected viral reservoir and an inflammatory site during chronic HIV and SIV infection. PLoS Pathog 2015; 11: e1005153

33. Lumeng CN, Bodzin JL, Saltiel AR. Obesity induces a phenotypic switch in adipose tissue macrophage polarization. J Clin Invest 2007; 117:175-84

34. Weisberg SP, McCann D, Desai M, Rosenbaum M, Leibel RL, Ferrante AW, Jr. Obesity is associated with macrophage accumulation in adipose tissue. J Clin Invest 2003; 112:1796-808

35. Gao D, Madi M, Ding C, Fok M, Steele T, Ford C, et al. Interleukin-1beta mediates macrophage-induced impairment of insulin signaling in human primary adipocytes. Am J Physiol Endocrinol Metab 2014; 307: E289-304

36. Lumeng CN, Deyoung SM, Saltiel AR. Macrophages block insulin action in adipocytes by altering expression of signaling and glucose transport proteins. Am J Physiol Endocrinol Metab 2007; 292: E166-74

37. Takemoto JK, Miller TL, Wang J, Jacobson DL, Geffner ME, et al. Insulin resistance in HIV-infected youth is associated with decreased mitochondrial respiration.AIDS 2017;31:15-23.

38. Qi Tangkai, Lu Hongzhou. AIDS-related metabolic syndrome and its treatment. World Clinics 2012; 33: $137-139$

\section{Tables}

Table 1. Baseline information of HIV infected male patients $(n=61)^{10-11}$ 


\begin{tabular}{|c|c|c|}
\hline viable & 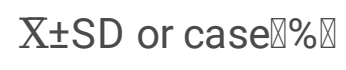 & range \\
\hline Age(yr】 & $32.05 \pm 8.38$ & $20 \rrbracket 58$ \\
\hline Infection duration(month) & $11.16 \pm 1.19$ & $1 \otimes 86$ \\
\hline \multicolumn{3}{|l|}{ T lymphocyte subsets } \\
\hline CD3+ count $($ cells/ul) & $1433.98 \pm 595.35$ & 470 3074 \\
\hline CD3+CD4+ count(cells/ul) & $313.87 \pm 118.473$ & $54 \sim 499$ \\
\hline CD3+CD4+ percentage $(\%)$ & $19.78 \pm 6.83$ & $1.40 \sim 43.40$ \\
\hline CD3+CD8+ count(cells/ul) & $1119.70 \pm 605.0$ & $360 \sim 2456$ \\
\hline CD3+CD8+ percentage $(\%)$ & $69.97 \pm 13.80$ & $36.13 \sim 97.20$ \\
\hline *Virus load of HIVRNA & $41772.77 \pm 10.38$ & $895.00 \sim 505987.00$ \\
\hline \multicolumn{3}{|l|}{ Glycolmetabolic parameters } \\
\hline FPG (mmol/L) & $5.50 \pm 0.508$ & $3.90 \sim 6.53$ \\
\hline $\mathrm{HbA1c}(\%)$ & $5.35 \pm 0.34$ & $4.5 \sim 5.9$ \\
\hline FINS (mIU/L) & $4.55 \pm 3.03$ & $0.5 \sim 11.09$ \\
\hline HOMA-IR (mlU*mmol/ $\left.\mathrm{L}^{2}\right)$ & $1.30 \pm 0.84$ & $0.81 \sim 2.31$ \\
\hline HOMA- $\beta$ (mIU/mmol) & $52.37 \pm 36.25$ & $14.8 \sim 182.46$ \\
\hline
\end{tabular}

Note: FPG, fasting plasma glucose; HbA1c, glycosylated hemoglobin; FINS, fasting insulin; HOMA-IR, hemeostasis model assement of insulin resistance; HOMA- $\beta$, hemeostasis model assement of $\beta$ cells. *refers to logarithmic transformation before statistical analysis for non-normally distributed data.

Table 2. Spearman correlation analysis of glucose metabolism parameters, general condition, anthropometric parameters, biochemical, immunological and virological indicators within 156 weeks $\square n=61 \rrbracket$ 


\begin{tabular}{|c|c|c|c|c|c|c|c|c|}
\hline & FPG & & FINS & & HOMA- & & HOMA & \\
\hline & $r$ & $\mathrm{p}$ & $r$ & $\mathrm{p}$ & $r$ & $\mathrm{p}$ & $r$ & $\mathrm{p}$ \\
\hline Age (year) & 0.120 & 0.008 & & & & & & \\
\hline $\begin{array}{l}\text { NAFLD } \\
\text { (0=without, } 1=\text { with) }\end{array}$ & 0.155 & 0.001 & & & & & & \\
\hline Follow-up weeks & & & 0.280 & 0.005 & & & & \\
\hline BMI $\triangle \mathrm{Kg} / \mathrm{m} 2)$ & 0.291 & 0.004 & & & & & & \\
\hline $\begin{array}{l}\text { Body fat } \\
\text { percentage } \otimes \% \bigotimes\end{array}$ & 0.311 & 0.002 & & & & & & \\
\hline $\begin{array}{l}\text { Body fat weight } \\
\text { \Kg囚 }\end{array}$ & 0.297 & 0.003 & & & & & & \\
\hline $\begin{array}{l}\text { Lean body mass } \\
\text { weight } ₫ \mathrm{Kg} \rrbracket\end{array}$ & 0.138 & 0.008 & & & & & & \\
\hline $\operatorname{ALT}(\mathrm{g} / \mathrm{L})$ & 0.126 & 0.006 & 0.221 & 0.031 & & & & \\
\hline AST $(g / L)$ & 0.081 & 0.029 & 0.352 & $<0.0001$ & & & & \\
\hline $\mathrm{GGT}(\mathrm{g} / \mathrm{L})$ & 0.127 & 0.005 & 0.230 & 0.025 & & & & \\
\hline $\operatorname{ALP}(g / L)$ & 0.106 & 0.004 & 0.253 & 0.009 & 0.164 & 0.002 & & \\
\hline $\mathrm{Cr}(\mu \mathrm{mol} / \mathrm{L})$ & 0.198 & $<0.0001$ & & & 0.378 & 0.027 & & \\
\hline Cysc (mg/L) & & & -0.280 & 0.005 & & & & \\
\hline $\mathrm{UA}(\mathrm{mmol} / \mathrm{L})$ & & & & & 0.175 & 0.001 & 0.179 & $<0.0001$ \\
\hline $\begin{array}{l}\text { CD3+ count (cells/ } \\
\mu l)\end{array}$ & -0.146 & $<0.0001$ & & & & & & \\
\hline $\begin{array}{l}\text { CD4+count (cells/ } \\
\mu \mathrm{l})\end{array}$ & -0.107 & 0.030 & & & & & & \\
\hline $\begin{array}{l}\text { CD8+count (cells/ } \\
\mu \mathrm{l})\end{array}$ & -0.120 & 0.002 & -0.236 & 0.022 & -0.121 & 0.026 & & \\
\hline CD3+\% (\%) & -0.140 & 0.002 & & & & & & \\
\hline $\mathrm{CD} 4+\%(\%)$ & & & 0.2363 & 0.023 & 0.111 & 0.042 & 0.118 & 0.030 \\
\hline CD8+\% (\%) & 0.134 & 0.007 & -0.293 & 0.004 & & & & \\
\hline CD4+/CD8+ & & & 0.312 & 0.002 & & & & \\
\hline $\lg M(g / L)$ & -0.263 & 0.003 & & & & & & \\
\hline LV (copes/L) & -0.266 & 0.033 & -0.374 & 0.045 & -0.427 & 0.024 & & \\
\hline CD4+ count groups & & & & & -0.340 & 0.049 & & \\
\hline
\end{tabular}


Abbreviations: ALP, alkaline phosphatase; ALT, alanine aminotransferase; AST, aspartate aminotransferase; $\mathrm{BMI}$, body mass index; $\mathrm{Cr}$, serum creatinine; Cysc, serum cystatin $\mathrm{C}$; FINS, fasting insulin; FPG, fasting plasma glucose; GGT, $\gamma$-glutamyl transpeptidase; HOMA-IR, hemeostasis model assement of insulin resistance; HOMA- $\beta$, the homeostasis model assessment of $\beta$ cell function; IgM, immunoglobulin M; NAFLD, non-alcoholic fatty liver disease; UA, uric acid; VL, viral load.

Table 3. Multiple stepwise regression analysis of risk factors of glucose metabolism parameters within 156 weeks $₫ n=61 \rrbracket$

\begin{tabular}{|c|c|c|c|c|c|c|}
\hline Independent variable & Dependent variable & B & Std.Erro & Beta & $t$ & $P$ \\
\hline \multirow[t]{3}{*}{ FPG } & (Constant) & 5.416 & 0.233 & - & 23.206 & $<0.0001$ \\
\hline & VL & -0.040 & 0.016 & -0.269 & -2.470 & 0.015 \\
\hline & $\mathrm{CD} 8+\%(\%)$ & 0.008 & 0.004 & 0.241 & 2.215 & 0.029 \\
\hline \multirow[t]{5}{*}{ FINS } & (Constant) & 83.179 & 27.700 & - & 3.003 & 0.007 \\
\hline & ALT & 0.348 & 0.081 & 0.670 & 4.302 & $<0.0001$ \\
\hline & VL & -6.654 & 2.375 & -0.734 & -2.801 & 0.011 \\
\hline & Follow-up weeks & -5.951 & 2.196 & -0.711 & -2.710 & 0.013 \\
\hline & CD8+ count (cells//ul) & -0.010 & 0.005 & -0.325 & -2.122 & 0.046 \\
\hline \multirow[t]{3}{*}{ HOMA-IR } & (Constant) & 18.025 & 7.686 & - & 2.345 & 0.029 \\
\hline & ALT & 0.090 & 0.023 & 0.642 & 3.855 & 0.001 \\
\hline & $\mathrm{VL}$ & -1.664 & 0.685 & -0.405 & -2.429 & 0.024 \\
\hline \multirow[t]{4}{*}{ HOMA- $\beta$} & (Constant) & 695.830 & 224.509 & - & 3.099 & 0.016 \\
\hline & ALT & 2.740 & 0.656 & 0.649 & 4.179 & $<0.0001$ \\
\hline & VL & -54.256 & 19.253 & -0.440 & -2.818 & 0.011 \\
\hline & CD8+ count $($ cells $/ / \mu \mathrm{l})$ & -0.090 & 0.039 & -0.345 & -2.271 & 0.034 \\
\hline
\end{tabular}

Abbreviations: ALT, alanine aminotransferase; FINS, fasting insulin; FPG, fasting plasma glucose; HOMAIR, hemeostasis model assement of insulin resistance; HOMA- $\beta$, the homeostasis model assessment of $\beta$ cell function; VL, viral load.

\section{Figures}



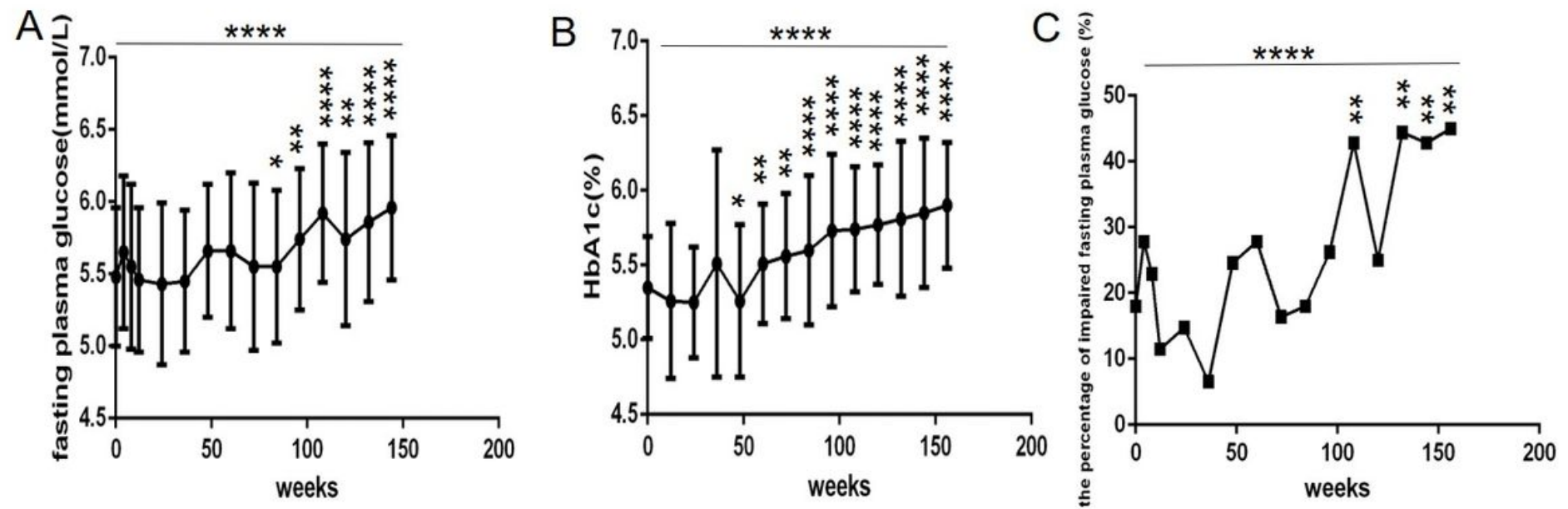

Figure 1

Long-term dynamic changes in FPG, $\mathrm{HbA} 1 \mathrm{c}$ and IFG within 156 weeks after the initiation of antiretroviral therapy with TDF plus 3TC plus EFV in male patients living with human immunodeficiency virus $(n=61)$. A. FPG level. B. HbA1c level C. IFG rate. Abbreviations: TDF, tenofovir. 3TC, lamivudine. EFV, efavirenz. FPG, fasting plasma glucose. IFG, impaired fasting plasma glucose. HbA1c, glycosylated hemoglobin A1c. ANOVA was used to compare glucose metabolism parameters from baseline to 156 weeks (A, B, C, all $P<0.0001)$. A paired t-test was used to compare glucose metabolism parameters between baseline and specific follow-up time points, ${ }^{*} \mathrm{P}<0.05,{ }^{*} \mathrm{P}<0.01, * * * * \mathrm{P}<0.0001$.
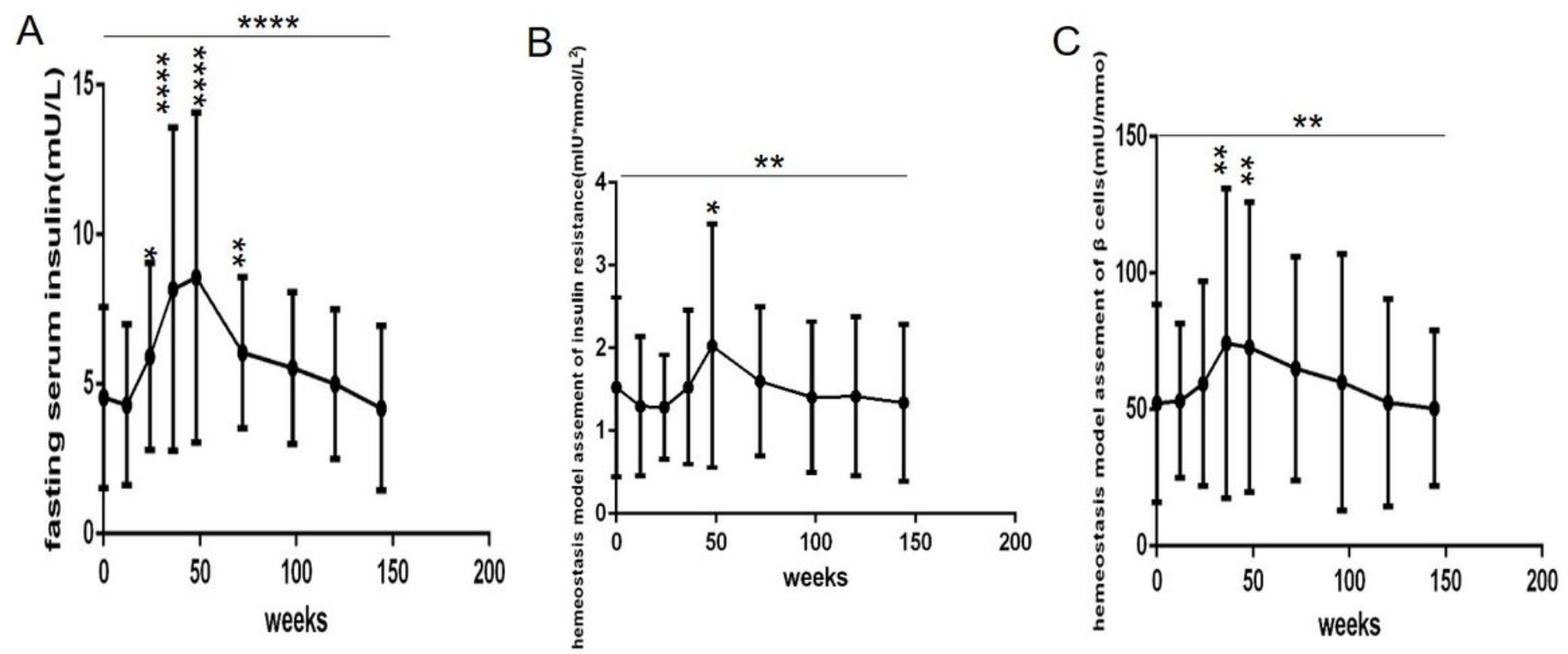

Figure 2

Long-term dynamic changes in other glucose metabolism parameters within 144 weeks after the initiation of antiretroviral therapy with TDF plus 3TC plus EFV in male patients living with human immunodeficiency virus $(n=61)$. A. FINS level. B. HOMA-IR value. C. HOMA- $\beta$ value. Abbreviations: TDF, tenofovir. 3TC, lamivudine. EFV, efavirenz. FINS, fasting serum insulin. HOMA-IR, homeostasis model 
assessment of insulin resistance. HOMA- $\beta$, Homeostasis model assessment of $\beta$ cell function. ANOVA was used to compare glucose metabolism parameters from baseline to 144 weeks $(A, P<0.0001 ; B, C$, all $P<0.01)$. A paired t-test was used to compare glucose metabolism parameters between baseline and specific follow-up time points, ${ }^{*} \mathrm{P}<0.05,{ }^{*} \mathrm{P}<0.01, * * \star * \mathrm{P}<0.0001$.
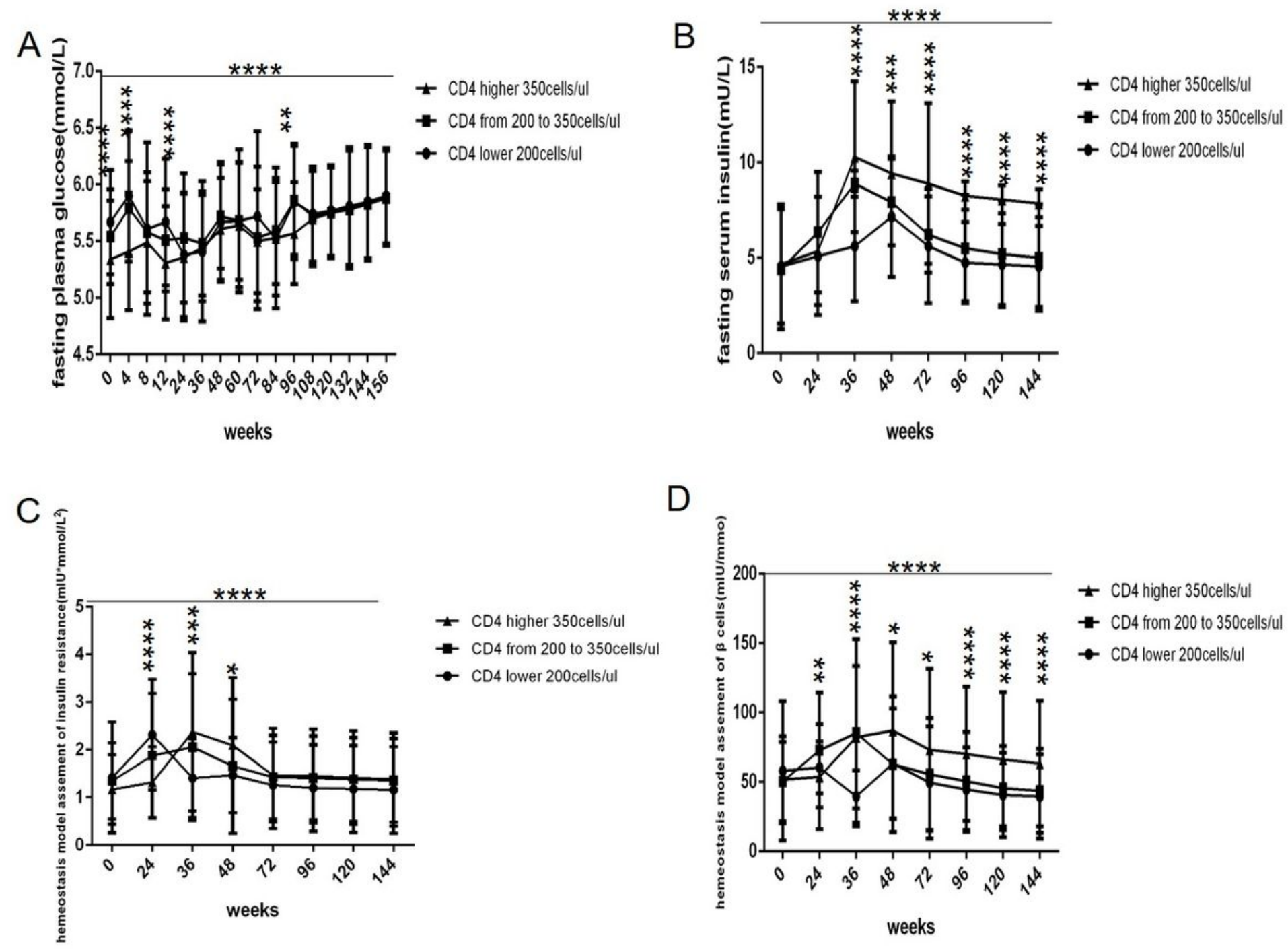

Figure 3

Long-term effects of baseline CD4+ T cell count on glucose metabolism parameters within 156 weeks after antiretroviral therapy with TDF plus 3TC plus EFV in male patients living with human immunodeficiency virus $(n=61 ; 26,12$, and 23 patients in the $<200$, from 200 to 350 , and $>350$ groups, respectively). A. FPG level. B. FINS level. C. HOMA-IR value. D. HOMA- $\beta$ value. Abbreviations: TDF, tenofovir. 3TC, lamivudine. EFV, efavirenz. FPG, fasting plasma glucose. FINS, fasting serum insulin. HOMA-IR, homeostasis model assessment of insulin resistance. HOMA- $\beta$, Homeostasis model assessment of $\beta$-cell function. Two-way ANOVA was used to compare glucose metabolism parameters among the three groups from baseline to 156 weeks (Interaction, $A, P<0.01 ; B, C, D$, all $P<0.0001$. Row factor, $A, B, C, D$, all $P<0.0001$. Column factor, $A, B, D$, all $P<0.0001 ; B, P<0.05)$. One-way ANOVA was used 
to compare glucose metabolism parameters among the three groups at the same time point, ${ }^{*} \mathrm{P}<0.05$, $\left.{ }^{\star *} \mathrm{P}<0.01,{ }^{* \star * *} \mathrm{P}<0.0001\right)$. 